\title{
On the multivalency of certain analytic functions
}

\author{
Mamoru Nunokawa ${ }^{1}$ and Janusz Sokół ${ }^{*}$
}

\footnotetext{
"Correspondence: jsokol@prz.edu.pl ${ }^{2}$ Department of Mathematics, Rzeszów University of Technology, Al. Powstańców Warszawy 12, Rzeszów, 35-959, Poland Full list of author information is available at the end of the article
}

\begin{abstract}
We prove several relations of the type $\left|\arg \left\{z f^{\prime \prime}(z) / f^{\prime}(z)\right\}\right| \leq\left|\arg \left\{f^{\prime}(z)\right\}\right|$ for functions satisfying some geometric conditions.

MSC: Primary 30C45; secondary 30C80

Keywords: analytic; univalent; multivalent; convex; starlike; strongly starlike; differential subordination
\end{abstract}

\section{Introduction}

Let $p$ be positive integer and let $\mathcal{A}(p)$ be the class of functions

$$
f(z)=z^{p}+\sum_{n=p+1}^{\infty} a_{n} z^{n}
$$

which are analytic in the unit disk $\mathbb{D}=\{z \in \mathbb{C}:|z|<1\}$ and denote $\mathcal{A}=\mathcal{A}(1)$.

The subclass of $\mathcal{A}(p)$ consisting of $p$-valently starlike functions is denoted by $\mathcal{S}^{*}(p)$. An analytic description of $\mathcal{S}^{*}(p)$ is given by

$$
\mathcal{S}^{*}(p)=\left\{f \in \mathcal{A}(p):\left|\arg \frac{z f^{\prime}(z)}{f(z)}\right|<\frac{\pi}{2}, z \in \mathbb{D}\right\} .
$$

The subclass of $\mathcal{A}(p)$ consisting of $p$-valently and strongly starlike functions of order $\alpha$, $0<\alpha \leq 1$ is denoted by $\mathcal{S}_{\alpha}^{*}(p)$. An analytic description of $\mathcal{S}_{\alpha}^{*}(p)$ is given by

$$
\mathcal{S}_{\alpha}^{*}(p)=\left\{f \in \mathcal{A}(p):\left|\arg \frac{z f^{\prime}(z)}{f(z)}\right|<\frac{\alpha \pi}{2}, z \in \mathbb{D}\right\} .
$$

The subclass of $\mathcal{A}(p)$ consisting of $p$-valently convex functions and $p$-valently strongly convex functions of order $\alpha, 0<\alpha \leq 1$, are denoted by $\mathcal{C}(p)$ and $\mathcal{C}_{\alpha}(p)$, respectively. The analytic descriptions of $\mathcal{C}(p)$ and $\mathcal{C}_{\alpha}(p)$ are given by

$$
\mathcal{C}(p)=\left\{f \in \mathcal{A}(p):\left|\arg \left\{1+\frac{z f^{\prime \prime}(z)}{f^{\prime}(z)}\right\}\right|<\frac{\pi}{2}, z \in \mathbb{D}\right\}
$$

and

$$
\mathcal{C}_{\alpha}(p)=\left\{f \in \mathcal{A}(p):\left|\arg \left\{1+\frac{z f^{\prime \prime}(z)}{f^{\prime}(z)}\right\}\right|<\frac{\alpha \pi}{2}, z \in \mathbb{D}\right\} .
$$

○ 2014 Nunokawa and Sokót; licensee Springer. This is an Open Access article distributed under the terms of the Creative Commons Attribution License (http://creativecommons.org/licenses/by/2.0), which permits unrestricted use, distribution, and reproduction in any medium, provided the original work is properly cited. 
For $p=1$ the classes $\mathcal{S}_{\alpha}^{*}(p)$ and $\mathcal{C}_{\alpha}(p)$ become the well known classes $\mathcal{S}_{\alpha}^{*}$ and $\mathcal{C}_{\alpha}^{*}$ of strongly starlike and strongly convex functions of order $\alpha$, respectively. The concept of strongly starlike and strongly convex functions of order $\alpha$ was introduced in [1] and [2] with their geometric interpretation. For $\alpha=1$ the classes $\mathcal{S}_{\alpha}^{*}$ and $\mathcal{C}_{\alpha}^{*}$ become the classes $\mathcal{S}^{*}$ and $\mathcal{C}^{*}$ of starlike and convex functions; see for example [3]. In this paper, we need the following lemma.

Lemma 1.1 Assume that $f \in \mathcal{A}$ with $f(z) / z \neq 0$ in $\mathbb{D}$. Assume also that for all $\theta, 0 \leq \theta<2 \pi$, the function $f$ satisfies the following condition:

$$
\begin{aligned}
\left(\mathfrak{I m} \frac{z f^{\prime \prime}(z)}{f^{\prime}(z)}\right) \sin \theta & =\rho\left(\frac{\mathrm{d} \arg \left\{f^{\prime}\left(\rho e^{i \theta}\right)\right\}}{\mathrm{d} \rho}\right) \sin \theta \\
& =\frac{1}{2} \mathfrak{R e}\left\{\frac{z f^{\prime \prime}(z)}{f^{\prime}(z)}(\bar{z}-z)\right\} \\
& =\frac{1}{2} \mathfrak{R e}\left\{\frac{f^{\prime \prime}(z)}{f^{\prime}(z)}\left(|z|^{2}-z^{2}\right)\right\} \geq 0,
\end{aligned}
$$

where $z=\rho e^{i \theta}, 0<\rho \leq r<1$. Then we have

$$
\left|\arg \left\{\frac{z f^{\prime}(z)}{f(z)}\right\}\right| \leq\left|\arg \left\{f^{\prime}(z)\right\}\right|, \quad z \in \mathbb{D} \text {. }
$$

Proof First we note that from

$$
0 \leq \arg \left\{z_{1}\right\} \leq \arg \left\{z_{2}\right\} \leq \pi \quad \Rightarrow \quad \arg \left\{z_{1}\right\} \leq \arg \left\{z_{1}+z_{2}\right\} \leq \arg \left\{z_{2}\right\},
$$

the implication

$$
0 \leq \arg \left\{z_{1}\right\} \leq \cdots \leq \arg \left\{z_{n}\right\} \leq \pi \quad \Rightarrow \quad \arg \left\{z_{1}\right\} \leq \arg \left\{\sum_{k=1}^{n} z_{k}\right\} \leq \arg \left\{z_{n}\right\}
$$

follows by mathematical induction.

For the case $0 \leq \theta<\pi, z=r e^{i \theta} \in \mathbb{D}$, we have

$$
\begin{aligned}
\arg \left\{\frac{f(z)}{z}\right\} & =\arg \left\{\frac{1}{r e^{i \theta}} \int_{0}^{r} f^{\prime}\left(\rho e^{i \theta}\right) e^{i \theta} \mathrm{d} \rho\right\} \\
& =\arg \left\{\int_{0}^{r} f^{\prime}\left(\rho e^{i \theta}\right) \mathrm{d} \rho\right\} .
\end{aligned}
$$

Let $0=\rho_{0}<\rho_{1}<\cdots<\rho_{n-1}<\rho_{n}=r, \Delta \rho_{k}=\rho_{k}-\rho_{k-1}, k=1, \ldots, n$. By $(1.1) \arg \left\{f^{\prime}\left(\rho e^{i \theta}\right)\right\}$ is an increasing function with respect to $\rho$, thus

$$
0=\arg \left\{f^{\prime}\left(\rho_{0} e^{i \theta}\right)\right\} \leq \arg \left\{f^{\prime}\left(\rho_{1} e^{i \theta}\right)\right\} \leq \cdots \leq \arg \left\{f^{\prime}\left(\rho_{n} e^{i \theta}\right)\right\}=\arg \left\{f^{\prime}\left(r e^{i \theta}\right)\right\} .
$$

Therefore, by (1.2) and by (1.4), we have

$$
\arg \left\{\sum_{k=1}^{n} f^{\prime}\left(\rho_{k} e^{i \theta}\right)\right\} \leq \arg \left\{f^{\prime}\left(r e^{i \theta}\right)\right\} \text {. }
$$


Using (1.5) in (1.3), we obtain

$$
\begin{aligned}
\arg \left\{\frac{f(z)}{z}\right\} & =\arg \left\{\int_{0}^{r} f^{\prime}\left(\rho e^{i \theta}\right) \mathrm{d} \rho\right\} \\
& =\arg \left\{\lim _{n \rightarrow \infty} \sum_{k=1}^{n} f^{\prime}\left(\rho_{k} e^{i \theta}\right) \Delta \rho_{k}\right\} \\
& =\lim _{n \rightarrow \infty} \arg \left\{\sum_{k=1}^{n} f^{\prime}\left(\rho_{k} e^{i \theta}\right) \Delta \rho_{k}\right\} \\
& \leq \arg \left\{f^{\prime}\left(r e^{i \theta}\right)\right\}
\end{aligned}
$$

or we have

$$
0 \leq \arg \left\{\frac{f(z)}{z}\right\} \leq \arg \left\{f^{\prime}(z)\right\}
$$

for $z=r e^{i \theta}$ and $0 \leq \theta \leq \pi$.

For the case $\pi \leq \theta<2 \pi$, from the hypothesis (1.1), we find that $\arg \left\{f^{\prime}\left(\rho e^{i \theta}\right)\right\}$ is an decreasing function with respect to $\rho$, thus

$$
0=\arg \left\{f^{\prime}\left(\rho_{0} e^{i \theta}\right)\right\} \geq \arg \left\{f^{\prime}\left(\rho_{1} e^{i \theta}\right)\right\} \geq \cdots \geq \arg \left\{f^{\prime}\left(\rho_{n} e^{i \theta}\right)\right\}=\arg \left\{f^{\prime}\left(r e^{i \theta}\right)\right\}
$$

and

$$
\arg \left\{\sum_{k=1}^{n} f^{\prime}\left(\rho_{k} e^{i \theta}\right)\right\} \geq \arg \left\{f^{\prime}\left(r e^{i \theta}\right)\right\}
$$

Therefore, in a similar way to above, we obtain

$$
\begin{aligned}
\arg \left\{\frac{f(z)}{z}\right\} & =\arg \left\{\int_{0}^{r} f^{\prime}\left(\rho e^{i \theta}\right) \mathrm{d} \rho\right\} \\
& \geq \arg \left\{f^{\prime}\left(r e^{i \theta}\right)\right\}
\end{aligned}
$$

and we also have

$$
0 \geq \arg \left\{\frac{f(z)}{z}\right\} \geq \arg \left\{f^{\prime}(z)\right\}
$$

for $z=r e^{i \theta}$ and $\pi \leq \theta \leq 2 \pi$. From (1.7) and (1.8), we have

$$
\begin{aligned}
\left|\arg \left\{\frac{z f^{\prime}(z)}{f(z)}\right\}\right| & =\left|\arg \left\{f^{\prime}(z)\right\}-\arg \left\{\frac{f(z)}{z}\right\}\right| \\
& \leq\left|\arg \left\{f^{\prime}(z)\right\}\right| .
\end{aligned}
$$

It completes the proof of Lemma 1.1. 
Corollary 1.2 Assume that $f \in \mathcal{A}$ with $f(z) / z \neq 0$ in $\mathbb{D}$. Assume also that $f(z)$ satisfies the following condition:

$$
\left(\mathfrak{I m} \frac{z f^{\prime \prime}(z)}{f^{\prime}(z)}\right) \mathfrak{I m}\{z\} \geq 0, \quad z \in \mathbb{D},
$$

then we have

$$
\arg \left\{\frac{z f^{\prime}(z)}{f(z)}\right\} \arg \{z\} \geq 0, \quad z \in \mathbb{D} .
$$

Proof The conditions (1.1) and (1.9) are equivalent. If $\arg \{z\} \geq 0$, then $z=r e^{i \theta} \in \mathbb{D}$ with $0 \leq \theta \leq \pi$. By (1.7), we have also

$$
\arg \left\{\frac{z f^{\prime}(z)}{f(z)}\right\} \geq 0, \quad z \in \mathbb{D} .
$$

If $\arg \{z\} \leq 0$, then $z=r e^{i \theta} \in \mathbb{D}$ with $\pi<\theta \leq 2 \pi$. By (1.8), we have also

$$
\arg \left\{\frac{z f^{\prime}(z)}{f(z)}\right\} \leq 0, \quad z \in \mathbb{D} .
$$

In both cases, we have (1.10).

The inequality (1.10) can be written in the equivalent form

$$
\left(\mathfrak{I m} \frac{z f^{\prime}(z)}{f(z)}\right) \mathfrak{I m}\{z\} \geq 0, \quad z \in \mathbb{D} .
$$

Recall that if $f(z)$ is analytic in $\mathbb{D}$ and $(\mathfrak{I m}\{f(z)\})(\mathfrak{I m}\{z\}) \geq 0$ in $\mathbb{D}$, then $f$ is called typically real function; see [4, Chapter 10]. Therefore, Corollary 1.2 says that if $z f^{\prime \prime}(z) / f^{\prime}(z)$ is a typically real function, then $z f^{\prime}(z) / f(z)$ is a typically real function, too.

\section{Main result}

Theorem 2.1 Let $f(z) \in \mathcal{A}$. Assume that for all $\theta, 0 \leq \theta<2 \pi, f(z)$ satisfies the following condition:

$$
\begin{aligned}
\left(\mathfrak{I m} \frac{z f^{\prime \prime}(z)}{f^{\prime}(z)}\right) \sin \theta & =\rho\left(\frac{\mathrm{d} \arg \left\{f^{\prime}\left(\rho e^{i \theta}\right)\right\}}{\mathrm{d} \rho}\right) \sin \theta \\
& \geq 0,
\end{aligned}
$$

where $z=\rho e^{i \theta}, 0 \leq \rho \leq r<1$, moves on the segment from $z=0$ to $z=r e^{i \theta}$ and

$$
\left|\arg \left\{f^{\prime}(z)\right\}\right| \leq \frac{\pi}{2}, \quad z \in \mathbb{D} .
$$

Then $f(z)$ is starlike in $\mathbb{D}$ or $f(z) \in \mathcal{S}^{*}$.

Proof From the hypothesis (2.1) and the hypothesis (2.2) and applying Lemma 1.1, we have

$$
\left|\arg \left\{\frac{z f^{\prime}(z)}{f(z)}\right\}\right| \leq\left|\arg \left\{f^{\prime}(z)\right\}\right| \leq \frac{\pi}{2}, \quad z \in \mathbb{D} .
$$

This shows that $f(z)$ is starlike in $\mathbb{D}$. 
Applying the same method as in the proof of Lemma 1.1, we have the following lemma.

Lemma 2.2 Let $f(z) \in \mathcal{A}(2)$. Assume that for all $\theta, 0 \leq \theta<2 \pi, f(z)$ satisfies the following condition:

$$
\begin{aligned}
\left(\mathfrak{I m} \frac{z f^{\prime \prime \prime}(z)}{f^{\prime \prime}(z)}\right) \sin \theta & =\rho\left(\frac{\mathrm{d} \arg \left\{f^{\prime \prime}\left(\rho e^{i \theta}\right)\right\}}{\mathrm{d} \rho}\right) \sin \theta \\
& \geq 0,
\end{aligned}
$$

where $z=\rho e^{i \theta}, 0 \leq \rho \leq r<1$ moves on the segment from $z=0$ to $z=r e^{i \theta}$. Then we have

$$
\left|\arg \left\{\frac{z f^{\prime \prime}(z)}{f^{\prime}(z)}\right\}\right| \leq\left|\arg \left\{f^{\prime \prime}(z)\right\}\right|, \quad z \in \mathbb{D} .
$$

Applying Lemma 2.2, we have the following theorem.

Theorem 2.3 Let $f(z) \in \mathcal{A}(2)$. Suppose that for all $\theta, 0 \leq \theta<2 \pi, f(z)$ satisfies the following condition:

$$
\begin{aligned}
\left(\mathfrak{I m} \frac{z f^{\prime \prime \prime}(z)}{f^{\prime \prime}(z)}\right) \sin \theta & =\rho\left(\frac{\mathrm{d} \arg \left\{f^{\prime \prime}\left(\rho e^{i \theta}\right)\right\}}{\mathrm{d} \rho}\right) \sin \theta \\
& \geq 0,
\end{aligned}
$$

where $z=\rho e^{i \theta}, 0 \leq \rho \leq r<1$, moves on the segment from $z=0$ to $z=r e^{i \theta}$ and

$$
\left|\arg \left\{f^{\prime \prime}(z)\right\}\right|<\frac{\pi}{2}, \quad z \in \mathbb{D} .
$$

Then we have $f(z) \in \mathcal{C}(2)=\mathcal{C}_{1}(2)$ or $f(z)$ is 2-valently convex in $\mathbb{D}$.

Proof From the hypothesis (2.3) and (2.4) and applying Lemma 2.2, we have

$$
\left|\arg \left\{\frac{z f^{\prime \prime}(z)}{f^{\prime}(z)}\right\}\right| \leq\left|\arg \left\{f^{\prime \prime}(z)\right\}\right|<\frac{\pi}{2}, \quad z \in \mathbb{D} .
$$

Therefore, we have

$$
1+\mathfrak{R e} \frac{z f^{\prime \prime}(z)}{f^{\prime}(z)}>0, \quad z \in \mathbb{D} .
$$

It completes the proof.

Applying the same method as in the proof of Lemma 1.1 and Lemma 2.2, we can generalize Theorem 2.1 and Theorem 2.3 as follows.

Lemma 2.4 Let $f(z) \in \mathcal{A}(p)$. Suppose that for all $\theta, 0 \leq \theta<2 \pi, f(z)$ satisfies the following condition:

$$
\begin{aligned}
\left(\mathfrak{I m} \frac{z f^{\prime \prime}(z)}{f^{\prime}(z)}\right) \sin \theta & =\rho\left(\frac{\mathrm{d}\left(\arg \left\{f^{\prime}\left(\rho e^{i \theta}\right)\right\}-(p-1) \theta\right)}{\mathrm{d} \rho}\right) \sin \theta \\
& =\rho\left(\frac{\mathrm{d} \arg \left\{f^{\prime}(z) / z^{p-1}\right\}}{\mathrm{d} \rho}\right) \sin \theta \\
& \geq 0,
\end{aligned}
$$


where $z=\rho e^{i \theta}, 0 \leq \rho \leq r<1$ moves on the segment from $z=0$ to $z=r e^{i \theta}$. Then we have

$$
\left|\arg \left\{\frac{z f^{\prime}(z)}{f(z)}\right\}\right| \leq\left|\arg \left\{\frac{f^{\prime}(z)}{z^{p-1}}\right\}\right|, \quad z \in \mathbb{D} .
$$

Proof For the case $0 \leq \theta \leq \pi$, from the hypothesis (2.5), we have

$$
\begin{aligned}
\arg \left\{\frac{f(z)}{z^{p}}\right\} & =\arg \left\{\frac{1}{r^{p} e^{i p \theta}} \int_{0}^{r} f^{\prime}\left(\rho e^{i \theta}\right) e^{i \theta} \mathrm{d} \rho\right\} \\
& =\arg \left\{\int_{0}^{r}\left|f^{\prime}\left(\rho e^{i \theta}\right)\right| e^{i\left(\arg \left\{f^{\prime}\left(\rho e^{i \theta}\right)\right\}-(p-1) \theta\right)} \mathrm{d} \rho\right\} \\
& \leq \arg \left\{f^{\prime}\left(r e^{i \theta}\right)\right\}-(p-1) \theta \\
& =\arg \left\{\frac{f^{\prime}(z)}{z^{p-1}}\right\}
\end{aligned}
$$

and therefore we have

$$
0 \leq \arg \left\{\frac{f(z)}{z^{p}}\right\} \leq \arg \left\{\frac{f^{\prime}(z)}{z^{p-1}}\right\}
$$

for $z=r e^{i \theta}$ and $0 \leq \theta \leq \pi$.

For the case $\pi<\theta<2 \pi$, applying the same method as above and in the proof of Lemma 1.1 and Lemma 2.2, we have

$$
0 \geq \arg \left\{\frac{f(z)}{z}\right\} \geq \arg \left\{\frac{f^{\prime}(z)}{z^{p-1}}\right\}
$$

for $z=r e^{i \theta}$ and $\pi<\theta<2 \pi$. From (2.6) and (2.7), we have

$$
\left|\arg \left\{\frac{z f^{\prime}(z)}{f(z)}\right\}\right|=\left|\arg \left\{\frac{f^{\prime}(z)}{z^{p}}\right\}-\arg \left\{\frac{f(z)}{z^{p}}\right\}\right| \leq\left|\arg \left\{\frac{f^{\prime}(z)}{z^{p-1}}\right\}\right| .
$$

It completes the proof of Lemma 2.4.

Thus, we have the following theorems.

Theorem 2.5 Let $f(z) \in \mathcal{A}(p)$. Assume that for all $\theta, 0 \leq \theta<2 \pi, f(z)$ satisfies the following condition:

$$
\begin{aligned}
\left(\frac{\mathrm{d}\left(\arg \left\{f^{\prime}\left(\rho e^{i \theta}\right)\right\}-(p-1) \theta\right)}{\mathrm{d} \rho}\right) \sin \theta & =\left(\frac{\mathrm{d} \arg \left\{f^{\prime}(z) / z^{p-1}\right\}}{\mathrm{d} \rho}\right) \sin \theta \\
& \geq 0,
\end{aligned}
$$

where $z=\rho e^{i \theta}, 0 \leq \theta<2 \pi, 0 \leq \rho \leq r<1$, moves on the segment from $z=0$ to $z=r e^{i \theta}$ and suppose that

$$
\left|\arg \left\{\frac{f^{\prime}(z)}{z^{p-1}}\right\}\right| \leq \frac{\alpha \pi}{2}, \quad z \in \mathbb{D}
$$

where $0<\alpha \leq 1$. Then we have $f(z) \in \mathcal{S}_{\alpha}^{*}(p)$ or $f(z)$ is $p$-valently and strongly starlike of order $\alpha$ in $\mathbb{D}$. 
Theorem 2.6 Let $f(z) \in \mathcal{A}(p), p \geq 2$. Assume that for all $\theta, 0 \leq \theta<2 \pi, f(z)$ satisfies the following condition:

$$
\begin{aligned}
\left(\mathfrak{I m} \frac{\left.z f^{\prime \prime \prime} z\right)}{f^{\prime \prime}(z)}\right) \sin \theta & =\rho\left(\frac{\mathrm{d}\left(\arg \left\{f^{\prime \prime}\left(\rho e^{i \theta}\right)\right\}-(p-2) \theta\right)}{\mathrm{d} \rho}\right) \sin \theta \\
& =\rho\left(\frac{\mathrm{d} \arg \left\{f^{\prime \prime}(z) / z^{p-2}\right\}}{\mathrm{d} \rho}\right) \sin \theta \\
& \geq 0,
\end{aligned}
$$

where $z=\rho e^{i \theta}, 0 \leq \rho \leq r<1$ moves on the segment from $z=0$ to $z=r e^{i \theta}$ and suppose that

$$
\left|\arg \left\{\frac{f^{\prime}(z)}{z^{p-2}}\right\}\right| \leq \frac{\alpha \pi}{2}, \quad z \in \mathbb{D}
$$

where $0<\alpha \leq 1$. Then we have $f(z) \in \mathcal{C}_{\alpha}(p)$ or $f(z)$ is $p$-valently and strongly convex of order $\alpha$.

Lemma 2.7 Let $f(z)=z+\sum_{n=2}^{\infty} a_{n} z^{n}$ be analytic in $|z| \leq 1$ and suppose that it satisfies the following condition:

$$
\mathfrak{R e}\left\{\frac{z f^{\prime \prime}(z)}{f^{\prime}(z)}\left(\overline{z e^{-i \alpha}}-z e^{-i \alpha}\right)\right\} \geq 0 \quad \text { in }|z| \leq 0,
$$

where $0 \leq \alpha \leq \pi$. Then for $\alpha \leq \theta \leq \alpha+\pi$ we have

$$
\begin{aligned}
\rho\left(\frac{\mathrm{d}\left(\arg \left\{f^{\prime}\left(\rho e^{i \theta}\right)\right\}\right)}{\mathrm{d} \rho}\right) & =\mathfrak{I m}\left\{\frac{\left.z f^{\prime} z\right)}{f(z)}\right\} \\
& \geq 0,
\end{aligned}
$$

while for $\alpha+\pi \leq \theta \leq \alpha+2 \pi$ we have

$$
\begin{aligned}
\rho\left(\frac{\mathrm{d}\left(\arg \left\{f^{\prime}\left(\rho e^{i \theta}\right)\right\}\right)}{\mathrm{d} \rho}\right) & =\mathfrak{I m}\left\{\frac{\left.z f^{\prime} z\right)}{f(z)}\right\} \\
& \leq 0,
\end{aligned}
$$

where $z=\rho e^{i \theta}, 0 \leq \rho \leq|z| \leq 1$.

Proof Let $z=\rho e^{i \theta}, 0 \leq \rho \leq|z| \leq 1$. Then it follows that

$$
\begin{aligned}
& \mathfrak{R e}\left\{\frac{z f^{\prime \prime}(z)}{f^{\prime}(z)}\left(\overline{z e^{-i \alpha}}-z e^{-i \alpha}\right)\right\} \\
& \quad=\mathfrak{R e}\left\{\frac{\mathrm{d} \log f^{\prime}(z)}{\mathrm{d} z}\left(\rho e^{-i(\theta-\alpha)}-\rho e^{i(\theta-\alpha)}\right)\right\} \\
& \quad=\mathfrak{R e}\left\{\rho\left(\frac{\mathrm{d} \log \left|f^{\prime}\left(\rho e^{i \theta}\right)\right|}{\mathrm{d} \rho}+i \frac{\mathrm{d} \arg f^{\prime}\left(\rho e^{i \theta}\right)}{\mathrm{d} \rho}\right)(-2 i)\right\} \sin (\theta-\alpha) \\
& \quad=2 \rho \frac{\mathrm{d} \arg f^{\prime}\left(\rho e^{i \theta}\right)}{\mathrm{d} \rho} \sin (\theta-\alpha) \\
& \quad \geq 0 .
\end{aligned}
$$


This proves (2.9) and (2.10) and it shows that the function $\arg f^{\prime}\left(\rho e^{i \theta}\right)$ is an increasing function with respect to $\rho, 0 \leq \rho \leq 1$, and $\alpha \leq \theta \leq \alpha+\pi$, and that the function $\arg f^{\prime}\left(\rho e^{i \theta}\right)$ is a decreasing function with respect to $\rho, 0 \leq \rho \leq 1$, and $\alpha+\pi \leq \theta \leq \alpha+2 \pi$.

Theorem 2.8 Let $f(z)=z+\sum_{n=2}^{\infty} a_{n} z^{n}$ be analytic in $\mathbb{D}$ and suppose that it satisfies the following condition:

$$
\mathfrak{R e}\left\{\frac{z f^{\prime \prime}(z)}{f^{\prime}(z)}\left(\overline{z e^{-i \alpha}}-z e^{-i \alpha}\right)\right\} \geq 0, \quad z \in \mathbb{D}
$$

where $0 \leq \alpha \leq \pi$ and

$$
\left|\arg \left\{f^{\prime}(z)\right\}\right| \leq \frac{\pi}{2}, \quad z \in \mathbb{D} .
$$

Then $f(z)$ is starlike in $\mathbb{D}$.

Proof From Lemma 2.7 and (2.12), for the case $0 \leq \theta \leq \pi$, we have

$$
\begin{aligned}
0 & =\left(\arg \frac{f(z)}{z}\right)_{z=0} \\
& \leq \arg \left\{\frac{1}{\rho e^{i \theta}} \int_{0}^{r} f^{\prime}\left(\rho e^{i \theta}\right) e^{i \theta} \mathrm{d} \rho\right\} \\
& =\arg \frac{f(z)}{z} \\
& =\arg \int_{0}^{r} f^{\prime}\left(\rho e^{i \theta}\right) \mathrm{d} \rho \\
& =\arg \int_{0}^{r}\left|f^{\prime}\left(\rho e^{i \theta}\right)\right| e^{i \arg \left\{f^{\prime}\left(\rho e^{i \theta}\right)\right\}} \mathrm{d} \rho \\
& \leq \arg \left\{f^{\prime}(z)\right\} .
\end{aligned}
$$

This shows that

$$
0 \leq \arg \left\{\frac{f(z)}{z}\right\} \leq \arg \left\{f^{\prime}(z)\right\},
$$

where $z=r e^{i \theta}, 0 \leq r<1$, and $\alpha \leq \theta \leq \alpha+\pi$.

For the case $\pi \leq \theta \leq 2 \pi$, applying the same method as above, we have

$$
0 \geq \arg \left\{\frac{f(z)}{z}\right\} \geq \arg \left\{f^{\prime}(z)\right\}
$$

where $z=r e^{i \theta}, 0 \leq r<1$, and $\pi+\alpha \leq \theta \leq 2 \pi+\alpha$. Applying (2.12), (2.13), and (2.14), we have

$$
\begin{aligned}
\left|\arg \left\{\frac{z f^{\prime}(z)}{f(z)}\right\}\right| & =\left|\arg \left\{f^{\prime}(z)\right\}-\arg \left\{\frac{f(z)}{z}\right\}\right| \leq\left|\arg \left\{f^{\prime}(z)\right\}\right| \\
& <\frac{\pi}{2}, \quad z \in \mathbb{D} .
\end{aligned}
$$

This completes the proof. 
Remark 2.9 The functions $f(z)=z+\alpha z^{2} / 2$ satisfy the conditions of Theorem 2.8 whenever $|\alpha| \leq 1 / 2$.

\section{Competing interests}

The authors declare that they have no competing interests.

\section{Authors' contributions}

All authors contributed equally to the writing of this paper. All authors read and approved the final manuscript.

\section{Author details}

'University of Gunma, Hoshikuki-cho 798-8, Chuou-Ward, Chiba, 260-0808, Japan. ${ }^{2}$ Department of Mathematics, Rzeszów University of Technology, Al. Powstańców Warszawy 12, Rzeszów, 35-959, Poland.

Received: 7 August 2014 Accepted: 3 September 2014 Published: 24 September 2014

\section{References}

1. Stankiewicz, J: Quelques problèmes extrêmaux dans les classes des fonctions $\alpha$-angulairement étoilées. Ann. Univ. Mariae Curie-Skłodowska, Sect. A 20, 59-75 (1966)

2. Brannan, DA, Kirwan, WE: On some classes of bounded univalent functions. J. Lond. Math. Soc. 1(2), 431-443 (1969)

3. Duren, PL: Univalent Functions. Springer, New York (1983)

4. Goodman, AW: Univalent Functions. Vols. I and II. Mariner Publishing Co., Tampa (1983)

doi:10.1186/1029-242X-2014-357

Cite this article as: Nunokawa and Sokót: On the multivalency of certain analytic functions. Journal of Inequalities and Applications 2014 2014:357.

\section{Submit your manuscript to a SpringerOpen ${ }^{\circ}$ journal and benefit from:}

- Convenient online submission

- Rigorous peer review

Immediate publication on acceptance

Open access: articles freely available online

- High visibility within the field

- Retaining the copyright to your article 